\title{
ENGEVISTA
}

Página da revista: http://www.uff.br/engevista/seer/

\section{Análise da adoção de medidores inteligentes como instrumento da política pública de eficiência energética}

\author{
Márcio Zamboti Fortes ${ }^{1}$ \\ Antônio Teixeira Ribeiro ${ }^{2}$ \\ David Anderson Veiga Gonçalves ${ }^{3}$ \\ Mariana Albuquerque Reynaud Schaefer ${ }^{4}$ \\ Milton Martins Flores ${ }^{5}$
}

Resumo: Neste artigo são discutidos os fatores que estimulam e inibem a adoção de medidores inteligentes no Brasil, apontando ainda porque estes dispositivos se tornaram um elemento central nas ações de redução de perdas afetando diretamente a eficiência dos sistemas de energia elétrica. Através de uma pesquisa exploratória na literatura foram mapeadas as políticas e estratégias adotadas nos EUA e Europa na implantação de smart grid para traçar um paralelo com as iniciativas em andamento no Brasil e analisar o papel desempenhado pelo medidor inteligente (smart meter) como elemento central de um projeto de smart grid. A análise das informações coletadas apontou que existem benefícios que são comuns aos projetos e iniciativas em andamento nessas regiões pesquisadas. Contudo, diferenças nas motivações e cenários socioeconômicos locais induzem a estratégias distintas, ainda que exista uma convergência nos objetivos principais de reduzir perdas e ampliar a oferta de energia de qualidade com redução das emissões de $\mathrm{CO}_{2}$.

Palavras-chave: Medição inteligente; eficiência energética; politica pública.

\footnotetext{
${ }^{1}$ UFF - Universidade Federal Fluminense

${ }^{2}$ UFF - Universidade Federal Fluminense

${ }^{3}$ UFF - Universidade Federal Fluminense

${ }^{4}$ UFF - Universidade Federal Fluminense

${ }^{5}$ UFF - Universidade Federal Fluminense
} 
ISSN: $1415-7314$

ISSN online: $2317-6717$

\begin{abstract}
This article discusses the factors that stimulate and inhibit the adoption of smart meters in Brazil, pointing also because these devices have become a central element in loss reduction actions directly affecting the efficiency of power systems. Through an exploratory research in the literature, it was mapped the adopted policies and strategies in the US and Europe in the smart grid deployment to draw a parallel with the initiatives underway in Brazil and to analyze the role played by the smart meter as a central element of a smart grid project. The analysis of information pointed out that there are benefits which are common to projects and initiatives underway in these areas surveyed. However, differences in motivations and local socioeconomic scenarios induce different strategies, although there is a convergence on key objectives to reduce losses and increase the quality of energy supply with reduced $\mathrm{CO}_{2}$ emissions.
\end{abstract}

Keywords: Smart metering, energy efficiency, public policy. 


\section{Introdução}

A humanidade tem experimentado nas últimas décadas uma evolução sem precedentes do bem-estar social. Ainda que essas melhorias não tenham atingido a todos de forma igualitária, o consumo per capita dos recursos naturais têm crescido numa taxa muito superior ao crescimento populacional (Goldemberg, 2007) colocando no centro do debate as questões relacionadas a eficiência, sustentabilidade e meio ambiente (Lopes, 2012).

Essas questões estão presentes também no setor elétrico marcado pelo desequilíbrio entre uma demanda crescente e limitação dos recursos não renováveis (Lopes, 2012). Como agravante, o sistema ainda convive com elevadas perdas decorrentes da infraestrutura em operação que é tecnologicamente defasada, pois pouco evoluiu nas áreas de automação, controle e comunicação. Entretanto, o advento das redes de computadores e da internet favoreceram o surgimento de um novo conceito: Redes Elétricas Inteligentes (Smart Grid) (Amin e Wollenberg, 2005).

Nesse artigo visamos analisar a motivação e estímulo à adoção de um dos elementos centrais do Smart Grid, o Smart Meter. Trata-se do dispositivo instalado na conexão entre o sistema elétrico e a rede elétrica do consumidor ou do prosumidor (Römer, 2012). A eletrônica embarcada nesse dispositivo permite coletar uma ampla variedade de informações e o seu compartilhamento através dos sistemas de comunicações e ainda uma atuação local com base em comandos remotos. Essas novas funcionalidades têm viabilizado, entre outros itens: a redução do custo operacional, redução das perdas técnicas e não técnicas e a inclusão no sistema de energia gerada a partir de fontes renováveis.

Adotou-se como metodologia de pesquisa a coleta de informações na literatura disponível. Numa segunda etapa foi identificado um conjunto de fatores que tem influência direta na formulação das estratégias para implantação do Smart Meter para finalmente proceder ao mapeamento e análise dessas informações que fundamentassem uma discussão e conclusões sobre as diferenças das estratégias adotadas.

\section{Desenvolvimento}

\subsection{Smart Grid}

Smart grid é um conceito que envolve múltiplas perspectivas: tecnológica, ambiental, socioeconômica, política e regulatória. A definição de smart grid pelos autores que cunharam o termo é de "uma infraestrutura de rede elétrica de larga escala caracterizada por segurança, agilidade e resiliência/robustez que enfrenta novas ameaças e condições não previstas" (Amin e Wollenberg, 2005). Segundo Lopes, "a ideia comum em smart grid é a integração da energia, de comunicações e tecnologias de informação para montar uma infraestrutura inteligente de energia elétrica que preveja uma evolução contínua de aplicações para benefício do usuário 
ISSN: $1415-7314$

ISSN online: $2317-6717$

final" (Lopes, 2012). Este modelo em adoção no mundo inteiro representa uma grande evolução do Sistema de Energia Elétrico abrangendo diversas inovações, tais como:

- Utilização de equipamentos eletrônicos como IED e smart meter, que abriram caminho para a automação, comunicação bi-direcional e intervenção remota (Gans, 2013);

- Uso da informação como um poderoso ativo para aprimorar a gestão operacional e o planejamento (Yang et al, 2009);

- Operação de fluxo de energia bi-direcional permitindo a transformação dos consumidores em prosumidores, ou seja, participantes do sistema elétrico que consomem e fornecem energia ao sistema (Römer, 2012);

- Fornecimento ao sistema elétrico de energia produzida a partir de fontes renováveis (Lopes, 2012).

A utilização de smart grid traz inúmeros benefícios para as empresas fornecedoras de energia elétrica e para a população em geral:

- Melhoria na qualidade da energia fornecida;

- Redução de perdas não técnicas em função da identificação mais precisa das fraudes e furtos, além de viabilizar o comando remoto (Depuru, 2011);

- Redução das perdas técnicas devido ao melhor controle da operação da rede, como distorção harmônica, níveis de tensão e corrente;

- Redução dos custos operacionais com leitura, corte, religação e outros ações que podem ser realizadas remotamente;

- Melhora da gestão de ativos;

- Custo evitado na expansão dos sistemas de geração e transmissão;

- Redução do consumo nos horários de pico em função da mudança do perfil de consumo de energia dos consumidores;

- Redução do consumo de energia em função do maior grau de informação do usuário;

- Possibilidade de introduzir outros tipos de tarifação;

- Redução de emissões de gases efeito estufa com a GD;

- Possibilidade de utilização de uma mesma infraestrutura de medição para outras unidades (água, gás etc.); e

- Integração com futuros sistemas de cidades inteligentes (Fortes, 2014).

\subsection{Smart Meter}

Os medidores de consumo de energia tradicionais, eletromecânicos ou eletrônicos, operam de forma isolada. Eles registram o consumo acumulado de energia elétrica ativa que, usualmente, é lido manualmente uma vez ao mês, disponibilizando a informação através de um 
processo rudimentar, oneroso e vulnerável a falhas. Na Figura 1 observa-se o fluxo de informação em um medidor tradicional.

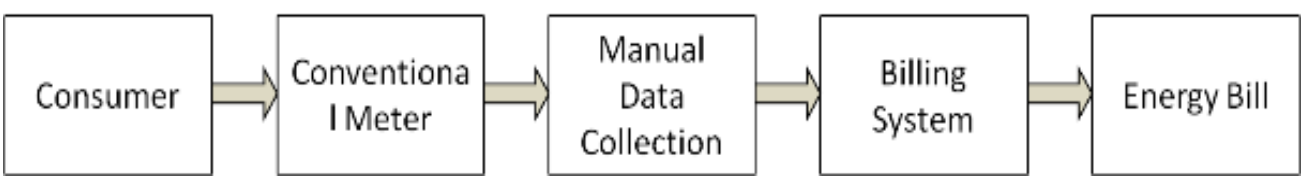

Figura 1. Fluxo de Informação em um Medidor Convencional

Os novos elementos integrados ao medidor lhe conferem a capacidade para capturar, processar, responder e comunicar novas informações. O compartilhamento dessas informações com os outros integrantes do sistema elétrico, como as unidades de geração de energia ou ainda elementos do sistema de transmissão e controle, propiciam, entre outras coisas, maior agilidade na identificação e solução de problemas operacionais e gestão mais eficiente do fluxo de energia, que passa a ser direcionado através de rotas mais confiáveis para os pontos que tem demanda a ser atendida.

Com a publicação da resolução 502/2012 da Agência Nacional de Energia Elétrica (ANEEL), foi regulamentado o uso dos medidores inteligentes para os consumidores do grupo B, consumidores residenciais. De acordo com esta resolução, é possível o consumidor escolher entre adoção de tarifa branca ou utilizar todas as funcionalidades que o smart meter pode proporcionar.

Os medidores inteligentes trazem diversos benefícios em relação aos medidores tradicionais. Suas principais funcionalidades são:

- Medição de energia ativa e reativa;

- Capacidade de aplicação de tarifas horárias;

- Demanda programável;

- Possibilidade de faturamento em pré-pagamento ou pós-pagamento eletrônico;

- Inversão de fluxo (geração distribuída);

- Registro de eventos e apuração de indicadores de continuidade e conformidade;

- Medição de neutro, sensor de abertura da tampa e alertas antifraude;

- Corte e religamento automático;

- Mostrador LCD parametrizável e display com seis dígitos;

- Saídas ou entradas de pulsos (ou saída serial) e porta ótica de comunicação local;

- Comunicação remota bidirecional

- Controle de demanda; entre outras. 


\subsection{Aspectos Econômicos e Regulatórios}

O investimento necessário para a implantação de uma infraestrutura moderna de medição (Advanced Metering Infrastructure - AMI) em uma unidade consumidora é alto se comparado à forma convencional. Porém, as vantagens de sua aplicação podem ser significantes para a sociedade, como por exemplo, melhoria na qualidade da energia, adiamento de investimentos na expansão da geração e transmissão entre outros. Neste item serão abordados os principais entraves para uma adoção massiva dos medidores inteligentes e serão apontadas algumas propostas de solução que vem sendo debatidas no setor.

\subsubsection{Aspectos Tarifários}

O sistema tarifário convencional brasileiro ainda traz um estimulo econômico para o consumidor adotar o smart meter. Porém está em discussão a implantação da Tarifa Branca, nela há valores diferenciados de tarifa para as horas e dias da semana, considerando momentos definidos como ponta ou fora de ponta. Para os sábados, domingos e feriados, seriam considerados como posto fora de ponta durante todo o dia. Para as demais situações, dias uteis, há divisões em três postos horários: Ponta, Intermediária e Fora de Ponta. A Figura 2 faz um comparativo entre as diferentes situações:

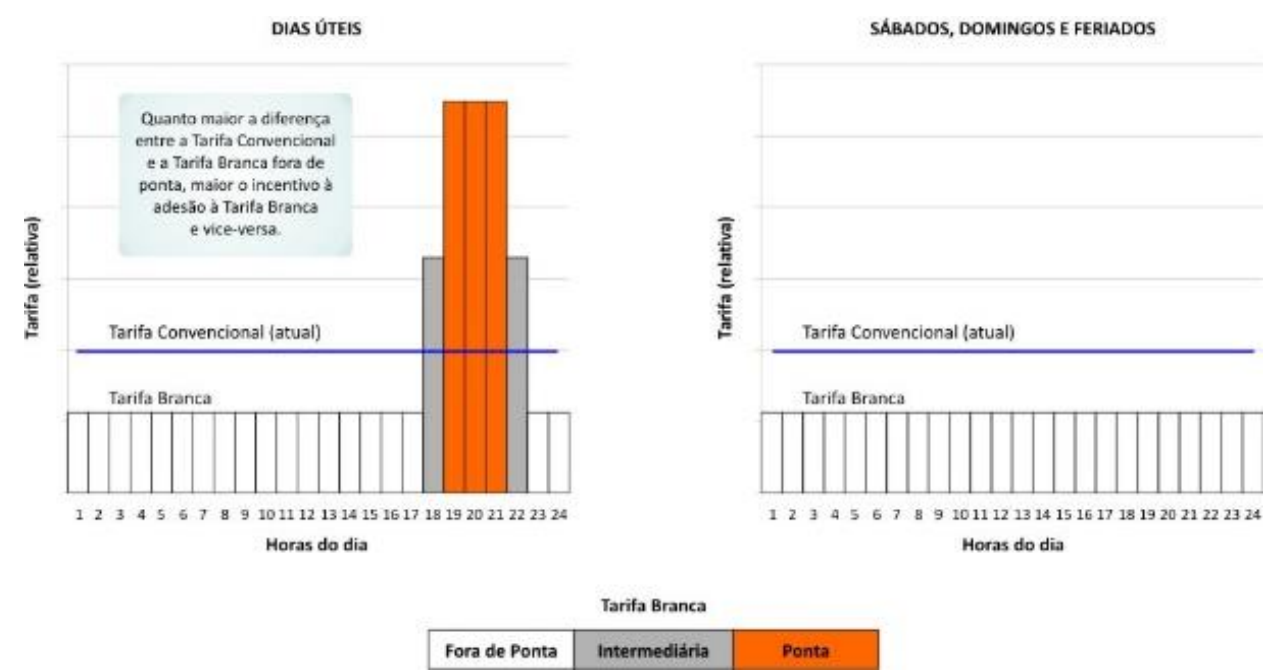

Figura 2. Comparativo entre Tarifas Branca e Convencional

A ideia é adotar a tarifa em caráter voluntário, mas se o consumidor adotar hábitos de consumo de energia que deem prioridade no período fora da ponta, consequentemente diminuindo o consumo na ponta e no intermediário, ele terá uma redução no valor pago pela energia consumida se comparada à modalidade convencional de tarifa, e isso poderia trazer um estimulo à adoção de tal tecnologia. Além do ganho econômico, o consumidor poderia se beneficiar de uma melhoria na qualidade do fornecimento de energia. Esse aumento na qualidade é potencialmente possível através do monitoramento em tempo real da rede que 
fornece com maior precisão os pontos que podem estar ocorrendo um defeito na rede, diminuindo o tempo de acionamento e de reparo das equipes de manutenção da concessionária.

Outra vantagem que este novo medidor (que é bidirecional) traria ao consumidor seria a possibilidade deste se tornar também um produtor, através de geração distribuída, podendo até vender seu excedente à concessionária.

\subsubsection{Politica Energética Integrada}

Pode-se perceber que os a implantação dos medidores inteligentes e de tarifas diferenciadas traz benefícios não só para os consumidores, mas também ao sistema elétrico como um todo. Com o monitoramento dos smart meter', a concessionária pode fazer um controle em tempo real do balanço de potência do seu sistema, possibilitando uma eficaz metodologia de combate às perdas comerciais.

Outra vantagem que a adoção desses esquemas permite é a possibilidade de um Gerenciamento pelo Lado da Demanda (GLD ou Demand Side Management - DSM), fazendo com que diversos objetivos possam ser atingidos na curva de carga do sistema, como por exemplo: redução de pico, deslocamento de cargas, entre outros, trazendo um benefício global para o Sistema Elétrico e para a população.

No entanto, nem todos esses ganhos são inteiramente capturados pelas distribuidoras de energia, logo, o nível de investimentos nesse segmento tenderá a ser menor do que um nível ótimo para a sociedade. Sendo assim, uma política energética que impulsione a disseminação das redes elétricas inteligentes é necessária para corrigir essas falhas no mercado. Configurando assim uma política energética integrada, mesclando eficiência energética, combate às perdas, energia renováveis, etc.

\subsubsection{Fontes de Recursos}

Um desafio imediato para tal Política Energética seria conseguir uma fonte de recursos para viabilizar a sua execução. O investimento necessário para a implantação de smart meter' numa unidade consumidora é muito maior se comparado à forma convencional. $\mathrm{O}$ Brasil tem cerca de 78 milhões de medidores para serem trocados. Mesmo que a universalização total dessa substituição não seja atingida, é um número desafiador.

Uma alternativa seria a criação de uma linha financiamento com juros baixos para fomentar a implantação dos medidores. Porém, alguns especialistas do setor veem na própria estrutura tarifária das distribuidoras uma possível oportunidade de recursos para esse objetivo. Essa alternativa seria remunerar de forma diferenciada os investimentos feitos em modernização da rede elétrica. 
Atualmente, a taxa de remuneração dos investimentos realizados pela distribuidora (Weighted Average Cost of Capital - WACC) é abatida de um fator, chamado Fator X, que consiste num índice de produtividade que ameniza o impacto inflacionário nos reajustes tarifários. Sendo assim, a proposta seria que esse Fator $\mathrm{X}$ fosse aumentado ou diminuído, conforme a menor ou maior aplicação dessas tecnologias pelas distribuidoras.

\subsubsection{Experiências Internacionais}

Na União Europeia (UE) a política para smart meter teve início através de medidas legislativas da UE com a Diretiva 96/92/CE do Parlamento Europeu, que estabeleceu regras comuns para todo o mercado de energia elétrica e deu partida ao smart grid e smart meter na Comunidade Europeia

A introdução dos smart meter' na UE teve como objetivo o monitoramento do consumo, a integração das fontes de energias renováveis, o incentivo a micro geração e a venda de energia pelos consumidores e prosumidores e a redução de emissões de $\mathrm{CO}_{2}$ e do consumo de energia, estimados na ordem de $9 \%$ e de até 3\%, respectivamente, com um investimento em torno de 45 bilhões de euros. Um relato dos investimentos da UE com smart meter' e projeções de retorno financeiro está descrito em Faruqui et al. (2010).

Na maioria dos países membros da UE já foram validadas as soluções em projetos pilotos, inclusive para as soluções de comunicação para o smart meter, em que foram considerados diversos padrões de comunicações com fio e sem fio, avaliados sobre os mais diversos critérios técnicos e não técnicos, sendo que não houve nenhum destaque entre as tecnologias, indicando para soluções mistas na maioria dos projetos implantados.

A fase atual é de disseminação dos sistemas de smart meter' com a previsão de implantação de 200 milhões de equipamentos até 2020 sendo que Itália, Suécia, Malta e Finlândia já atingiram as metas antes dos prazos, e em alguns países tais como Bélgica, República Tcheca e Lituânia os estudos de custo-benefício foram negativos para a implantação dos medidores inteligentes. A maior parte das regulamentações nacionais estabelece normas de conduta e atribui que a responsabilidade pelos medidores seja das distribuidoras de energia. $\mathrm{O}$ Reino Unido é uma exceção, pois possui um dos mercados de energia mais desregulamentado do mundo, sendo a responsabilidade da medição de empresas varejistas de energia (retailer) e o cliente é o único que vai decidir sobre a implantação da medição inteligente (Erlinghagen et al, 2015). A diretiva 2009/72/EC, que trata da modernização das redes de distribuição de energia elétrica e da introdução das redes inteligentes, estabeleceu um tríplice objetivo 20-20-20, com $20 \%$ de energia de fontes renováveis, redução de $20 \%$ nas emissões de $\mathrm{CO}_{2}$ e crescimento de $20 \%$ na produção de energia como meta no ano de 2020.

Nos Estados Unidos, o Departamento de Energia (DOE) desenvolve a coordenação nacional, com parcerias com a indústria, os meios acadêmicos e os governos estaduais, 
existindo uma grande independência entre as partes. O marco inicial do processo ocorreu no ano de 1997, através do "Energy Independence and Security Act of 2007", que deu partida para o smart grid e smart meter, com o objetivo de modernizar a infraestrutura de distribuição de energia elétrica, tornar a rede mais segura e mais confiável, principalmente considerando os grandes apagões que sistematicamente vinham ocorrendo, facilitar o crescimento das fontes de energia renováveis e melhor gerenciar o consumo e os custos da energia e o viés político de manter os Estados Unidos como líder global.

Em 2013 os Estados Unidos possuíam em torno de 50 milhões de medidores inteligentes instalados, dos quais $89 \%$ eram usuários residenciais e projeção de 65 milhões em 2015

Vários estados aprovaram regulamentação sobre fontes renováveis, entre os quais o importante estado da Califórnia aprovou regulamentação que exige 33\% de energia fornecida de fontes renováveis até 2020, destacando a energia fotovoltaica, que embora represente $0,2 \%$ do consumo de energia, tem crescimento acelerado da ordem de $50 \%$ ao ano. Um estudo do National Renewable Energy Laboratory (NREL) prevê que para 2035 em torno de $80 \%$ do fornecimento de energia será de fontes renováveis.

Para a política americana baseada na nova geração de consumidores e prosumidores, e em mini e micro geradores, é necessária a disseminação de smart meter' para o fornecimento de dados para a avaliação de variáveis domésticas, tais como a segmentação, planejamento e comunicação, de maneira a permitir ações de eficiência energética para a eficácia dos programas de nível federal, estadual e local. (Kavousian, 2015).

O Brasil já possui uma matriz de energia elétrica relativamente limpa que utiliza recursos de energia renováveis (Goldemberg, 2007), sendo a disseminação de smart meter para o grupo "B" de consumidores uma estratégia para a gestão do consumo, inclusão das novas fontes de energia e de novos tipos de faturamento. Além dos projetos pilotos, a política governamental ou das distribuidoras de energia para rollout dos medidores é ainda principiante e focada no combate ao furto e na inadimplência (Leite, 2013). Para corroborar esta afirmação a Resolução Normativa 502, que determinava a adoção de medidores inteligentes pelas concessionárias em até 18 meses após a sua publicação, foi suspensa.

Existem alguns projetos pilotos acontecendo no Brasil que podem contribuir para a criação de uma política adequada a implantação massiva desta nova tecnologia, cita-se o exemplo da Cidade de Búzios, no Rio de Janeiro. (Fortes et al.,2014)

\section{Conclusão}

Neste artigo foi apontado o desafio em equilibrar a demanda crescente por energia em um mundo ainda muito dependente das fontes de energia não renováveis. Pôde-se perceber que 
o crescimento da demanda por energia elétrica ocorre com base em dois vetores: crescimento demográfico e crescimento do consumo per capita de energia, resultando num crescimento exponencial da demanda por energia elétrica.

Com base nos dados, foram consideradas três linhas de ação para equilibrar a relação entre produção e consumo de energia elétrica:

I) Aumento da eficiência do ciclo de produção, que envolve também o transporte e distribuição de energia, ou seja, reduzindo as perdas em geral e aprimorando a conversão de outras formas de energia em energia elétrica;

II) Aumento da eficiência em todas as formas de consumo de energia elétrica, seja na transformação da energia em trabalho útil ou na alocação espaço-temporal da energia, sem que isso afete o crescimento do bem estar social e o crescimento da atividade econômica;

III) Alteração drástica da matriz energética que deve tornar-se preponderantemente baseada em fontes de energia renováveis.

Tanto o aumento da eficiência quanto o aumento da oferta de energia produzida por fontes renováveis dependem da implementação do smart meter, que agrega inteligência ao Sistema Elétrico, transformando-o em um smart grid. Suas funções combinadas de coleta de dados, comunicação e atuação remota, permitem monitorar e controlar a qualidade e quantidade da energia entregue ao consumidor, tornando possível identificar as perdas técnicas e não técnicas no sistema elétrico e, portanto, adotar ações para melhorar a eficiência do sistema.

O smart meter em conjunto com as tarifas diferenciadas, como a tarifa branca ou TOE, induzem a mudança na curva de carga dos consumidores, migrando o consumo do horário de pico para os horários de menor demanda tornando possíveis investimentos em função do uso otimizado da infraestrutura já disponível.

A condição de monitoração em tempo real da demanda e oferta de energia proporcionada pelo smart meter, permite às operadoras identificarem a necessidade para ajustar o grid se for necessário para compensar as distorções introduzidas no sistema com a entrada e saída de operação das fontes de energia renováveis. A ausência dessas ações inviabilizaria o aproveitamento das fontes de energia renováveis em pequena escala que tem um regime de operação irregular e dependente do clima.

A Comunidade Europeia e EUA se esforçam em aumentar a participação das fontes de energia renováveis na sua matriz energética, adotando medidas para estimular a produção de energia em microgrids e incentivar os consumidores a consumir de forma mais eficiente. $\mathrm{O}$ modelo de produção descentralizado e implantação de smart meter desempenham um papel importante nessa estratégia, justificando os elevados investimentos na instalação de milhões de 
smart meter e unidades de pequeno ou médio porte de produção de energia a partir, principalmente, de sistemas eólicos e sistemas fotovoltaicos.

O Brasil mantém sua tradição em investir na construção de grandes usinas hidrelétricas, garantindo uma posição mais confortável quanto a participação de energias renováveis na sua matriz elétrica. Programas que fomentam a eficiência energética têm sido desenvolvidos, como o PROCEL com foco no segmento residencial e no segmento de grandes consumidores. Contudo, as iniciativas para implantação do smart grid no Brasil não têm obtido prioridade na agenda dos agentes públicos, que necessitam assumir o papel de coordenadores da transformação do sistema elétrico num modelo segundo o conceito de smart meter. A transformação do sistema elétrico convencional em smart grid é inquestionável, mas não há um participante com incentivo suficiente para liderar esse processo, tornando necessário que um agente público assuma essa liderança, usando os instrumentos regulatórios, como já fizeram a comunidade europeia e EUA, para: criar estímulos tarifários, como a tarifa branca; fomentar a área de pesquisa para alcançar independência tecnológica e gerar novos negócios, e redistribuir custos e retornos entre os agentes econômicos, para estimular uma mudança que beneficiará toda a sociedade.

\section{Referências}

AMIN,S.M.; WOLLENBERG,B.F.Toward a smart grid: Power delivery for the $21^{\text {st }}$ century. IEEE Power and Energy Magazine, v.3, n.5, p.34-41, 2005.

DEPURU, S.S. ; WANG,L. ; DEVABHAKTUNI, V. Electricity theft: overview, issues, prevention and a smart meter based approach to control. Energy Policy, v.39, p.1007-1015, 2011.

FARUQUI,A.; HARRIS,D.; HLEDIK, R. Unlocking the $€ 53$ billion savings from smart meters in the EU: How increasing the adoption of dynamic tariffs could make or break the EU's smart grid investment. Energy Policy, v.38, p.6222-6231, 2010.

FORTES, M.Z. et al. Smart City Búzios - Experiência para a Expansão de Sistemas de Geração com Fontes Alternativas. In: IX Congresso Brasileiro de Planejamento Energético, 2014.

GANS,W.; ALBERINI,A.; LONGO,A. Smart meter devices and the effect of feedback on residential electricity consumption: Evidence from a natural experiment in Northern Ireland. Energy Economics, v.36, p.729-743, 2013.

GOLDEMBERG, J.; LUCON,O. Energias renováveis: um futuro sustentável. Revista USP, v.72, p .6-15, 2007.

KAVOUSIAN, A.; RAJAGOPAL, R.; FISCHER,M. Ranking appliance energy efficiency in households: Utilizing smart meter data and energy efficiency frontiers to estimate and identify the determinants of appliance energy efficiency in residential buildings. Energy and Buildings, v.99, p.220-230, 2015.

LEITE,D.R.V. Medidores eletrônicos: Análise de viabilidade econômica no contexto de redes inteligentes. Dissertação de Mestrado em Engenharia Elétrica, Universidade de Brasilia, 2013.

LOPES,Y. et al. Smart Grid e IEC 61850: Novos Desafios em Redes e Telecomunicações para o Sistema Elétrico, In: Proceedings of SBrT'2012, Mini-Cursos, 2012.

ROMER,B. et al. The role of smart metering and decentralized electricity storage for smart grids: The importance of positive externalities, Energy Police, v.50, p.486-495, 2012. 
ISSN: $1415-7314$

ISSN online: $2317-6717$

ERLINGHAGEN,S.; LICHTENSTEIGER, B.; MARKARD, J. Smart Meter communication standards in Europe - a comparision. Renewable and Sustainable Energy Reviews. v.43, p.1249-1262, 2015.

YANG,Z.; LEE,W.K; LAM,H.Y. Collaboration Interface in Smart Metering Scheme, In: The International Confereence on Electrical Engineering, p.1-6, 2009. 